\title{
To investigate the Association between Normative \\ Commitment (Sense of Obligation to Stay) and Employee's Performance in "FENDA COMMUNICATION AND IT -KSA"
}

\author{
Dr. Rafia Gulzar \\ Assistant Professor \\ Dar Al Uloom University \\ Al Falah Area- 3535, Saudi Arabia
}

Received: Aug. 8, $2021 \quad$ Accepted: Aug. 26, $2021 \quad$ Online published: Sep. 7, 2021

doi:10.5296/ijhrs.v11i3.18994 URL: https://doi.org/10.5296/ijhrs.v11i3.18994

\begin{abstract}
Normative commitment happens when employee feel a sense of obligation towards his organization, even if employee is not happy with tasks and responsibility assigned, or even if employee desires to chase better chances or opportunity. Normative commitment(NC) is one of the three components of Organizational commitment that has received less attention in research than other two components ( Affective and Continuance).The main purpose of this research paper was to critically understand the normative commitment and evaluate the existing relationship between normative commitment and employees performance in "FENDA COMMUNICATION AND IT -KSA". The Total number of respondents were 68 surveyed and eight statements used to measure the normative commitment, has been developed by Allen and Meyer (1991) and three -statements used for measuring employee performance has been developed by the Williams and Anderson (1991). The data was analyzed that clearly showed that there is a close relationship between normative commitment and employees' performance in sample study organization.
\end{abstract}

Keywords: affective commitment, continuance commitment, employee commitment, employees job performance, normative commitment

\section{Introduction}

In order to compete in the global market Saudi Arabia is reforming its economy to familiarize new development in both public and private sector. Organizations that attempt to achieve 
high market share on national and international level need to focus on low rate of employee turnover and high employee commitment. Employee commitment is highly valuable. Studies have highlighted that commitment has a great impact on the successful performance of an organization. The great Scholar Meyer and Allen (1990) of organizational commitment advocate three types of organizational commitment that are, affective commitment which measures an employee's emotional attachment to, identification with, and involvement in the organization, normative commitment reflects pressures on an employee to remain with an organization resulting from organizational obligations. Continuance commitment (CC) refers commitment associated with the costs that employees perceive are related to leaving the organization. Commitment of employees can be an important instrument for improving the performance of the organizations. The higher level of communication in organization led to higher level of commitment and there by increased performance (Chen, Silverthorne and Hung 2006).

Researchers indicates that many studies are available in developed countries for understanding the relationship between job performance and Organizational commitment. However, less work is available to know the association between organizational commitment and job performance in Middle east especially in Saudi Arabia. Keeping this in mind this study considered one element i.e., Normative commitment out of three components of organizational commitment were taken for study purpose. Hence, this study will begin with understanding the Normative commitment and will also attempt to explore the relationship between in Normative commitment and employee performance within the FENDA Communication and IT-KSA.

\section{Literature Review}

\subsection{Normative Commitment}

According to Meyer et al., (2002) NC is characterized by a mindset of obligation (e.g., obligation to remain with the organization or support a change initiative). Although theoretically distinct from AC (mindset of desire) and CC (mindset of cost-avoidance). In the 1980s, Wiener and his colleagues conducted a series of studies based on what they described as a "normative view" of organizational commitment (Vardi, Wiener, \& Popper, 1989; Wiener, 1982; Wiener \& Vardi, 1980). Wiener (1982, p. 421) defined commitment as reflecting "the totality of internalized normative pressures to act in a way that meets organizational goals and interests" and argued that committed employees are willing to make personal sacrifices for the sake of the organization because they "believe it is the "right' and moral thing to do." This contrasts with instrumental motivation where behavior is guided more by an evaluation of personal costs and benefits. According to Wiener, (normative) commitment develops as the result of both cultural/familial and organizational socialization processes. Like Wiener, Scholl (1981) argued that commitment must be clearly distinguished from instrumental motivation. He defined commitment as "a stabilizing force that acts to maintain behavioral direction when expectancy/equity conditions are not met and do not function”, Meyer and Allen (1991) noted that they reflected a common theme of obligation. Given a general agreement that this sense of obligation derives from the internalization of normative influences, they adopted the 
term "normative commitment." They argued that "employees with strong normative commitment will remain with an organization by virtue of their belief that it is 'the right' and moral thing to do". More recently, Meyer and Herscovitch (2001) proposed that the TCM can be applied to other workplace commitments and defined NC more generally as "the mindset that one has an obligation to pursue a course of action of relevance to a particular target". In their application of this model to organizational change initiatives, Herscovitch and Meyer (2002) defined NC as "a sense of obligation to provide support for the change". Normative commitment is referred to as obligatory commitment to the organization (Setti, 2014). Normative commitment focuses on the individual's sense of obligation to stay with the organization. This commitment stems from an individual's moral obligation to stay with the organization regardless of the benefit he or she might receive by leaving (Radosavljevic, Cilerdzic \& Dragic, 2017).

\subsection{Employees Performance}

Employee performance is among the critical factors that contribute significantly in organizational success. Employee performance is defined as how an employee fulfills their job duties and executes their required tasks. It refers to the effectiveness, quality, and efficiency of their output. Performance also contributes to our assessment of how valuable an employee is to the organization. Dharma (1991), thought that the performance is somewhat that is prepared, or products shaped and offered by a cluster of people. Employees performance also depends on their internal satisfaction towards their job. If employees are satisfied from their jobs as well as the organization than they are more keenly interested to perform well towards organizational goal achievement (Harter et al, 2002). Prawirosentoso (2000), explored that performance is outcome of work in an efficient way with considerable obligation for organization without interrupting any law and organizational goals. According to Y1ldiz, Savc1, and Kapu (2014), work performance defined as the fulfillment or completion of the work is the success level of making efforts that employees can perform their works. Bingöl (2003), on the other hand, defines performance as the execution of work according to the given conditions or as identifying employees' behavior.

\subsection{Association Between Normative Commitment and Employees Performance}

Organizational commitment has been linked both theoretically and empirically to individual performance. Meyer and Allen (1997) have argued that both normative and affective commitment will be linked performance, whereas continuance commitment will be unrelated or even negatively related.

The research carried out by Uygur (2007), on bank employees demonstrated that there was a positive relationship between organizational commitment and employee performance. The research of Özutku (2008), done on factory workers revealed that there was a positive and significant relationship between affective commitment and continuance commitment and work performance, but there wasn't a significant relationship between normative commitment and work performance. Dixit and Bhati (2012), found that the Employees Commitment (Affective, Normative, continuous) were significantly associated with sustained productivity in Auto component industry. They reported that there were positive relationships 
between the three commitments (affective, continuance and normative commitment) and sustained productivity of the organization. Qaisar et al. (2012), discussed about the effects of organizational commitment on employee's performance. They examined the effect of three components of organizational commitment (affective, continuance and normative commitment) on performance of police officers in Pakistan. The study of Iraz and Akgün (2011), performed on bank employees displayed that there was a positive relationship between normative commitment and performance.

Based on the above literature, the following hypothesis were examined:

H1: Normative commitment has positive association with employees work performance.

\section{The Research Methodology}

The present study is carried to achieve main objective to understand the concept of normative commitment and its association with employee's performance. The research method is survey and statistical method is descriptive. Using survey method or field research is an ordered information collecting method that can be used for detecting relationship between various variables.

\subsection{Sample}

In this study, statistical population include both managerial $84 \%$ and non-managerial $16 \%$ staff of FENDA communication and IT -KSA Riyadh. This research study considers employees both male (82\%) and female (18\%) in the selected sample organization (FENDA Communication and IT -KSA.). The total number of respondents were 68. Actually, 80 questionnaires were distributed and in return, only 68 were useful for analysis purpose so response rate was $85 \%$. The questionnaire used in this study has 8 -items which were verified on 5-likert scale, 5=strongly agree and 1=strongly disagree to measure normative commitment based on Meyer and Allen (1997) questionnaire. In order to measure employee job performance of FENDA Communication and IT -KSA three items were selected, from the questionnaire designed by William and Anderson (1991) high score on the selected items indicates high performance and low score predicts low performance of an employees.

\subsection{Validity and Reliability of Questionnaire}

This study has used the standardized questionnaires of Allen and Meyer organizational commitment (1991) and William and Anderson (1991).

\section{Exhibit 1:1The reliability of measuring instruments}

\begin{tabular}{|l|l|l|l|l|}
\hline S. No & Variables & $\begin{array}{l}\text { No. of } \\
\text { items }\end{array}$ & $\begin{array}{l}\text { Cronbach's Alpha } \\
(\alpha)\end{array}$ & $\begin{array}{l}\text { Guttman } \\
\text { coefficient }\end{array}$ \\
\hline 1. & $\begin{array}{l}\text { Normative } \\
\text { commitment }\end{array}$ & 8 & 0.79 & 0.78 \\
\hline 2. & $\begin{array}{l}\text { Employees job } \\
\text { performance. }\end{array}$ & 3 & 0.91 & 0.89 \\
\hline
\end{tabular}

These questionnaires have been used in several studies and therefore, the used measurement instruments in this study was properly validated, and in fact, its validity has been obtained via 
the content of it. In this study, the reliability coefficients were calculated by using of both Cronbach's alpha and Guttman spilt-half coefficient as shown in the above exhibit 1.1.

\section{Research Findings}

The current study was carried to test the hypothesis to understand the relationship between Normative Commitment with employee job performance in the sample study organization. The results are examined and debated in the following tables:

Table 1 depicts Normative Commitment NC1: "I think that people these days move from company to company too often." with Employees performance (EP) like I feel my performance is getting improved due to my fulfillment (EP1), My result is getting well due to positive ness (EP2), Employee understand strategies and are committed towards goals and result (EP3) and Employee job Performance(EP) overall. It is found that 18(26.4\%) Agree with NC1. Among them $88 \%$ A ,76\% SA, 80\% A and 92\%A with EP1, EP2, EP3 and EP overall, respectively and are statistically significant at $\mathrm{p}<0.05$.

Table 1. Depicts the Association of Normative Commitment NC1 "I think that people these days move from company to company too often." with Employees performance (EP)

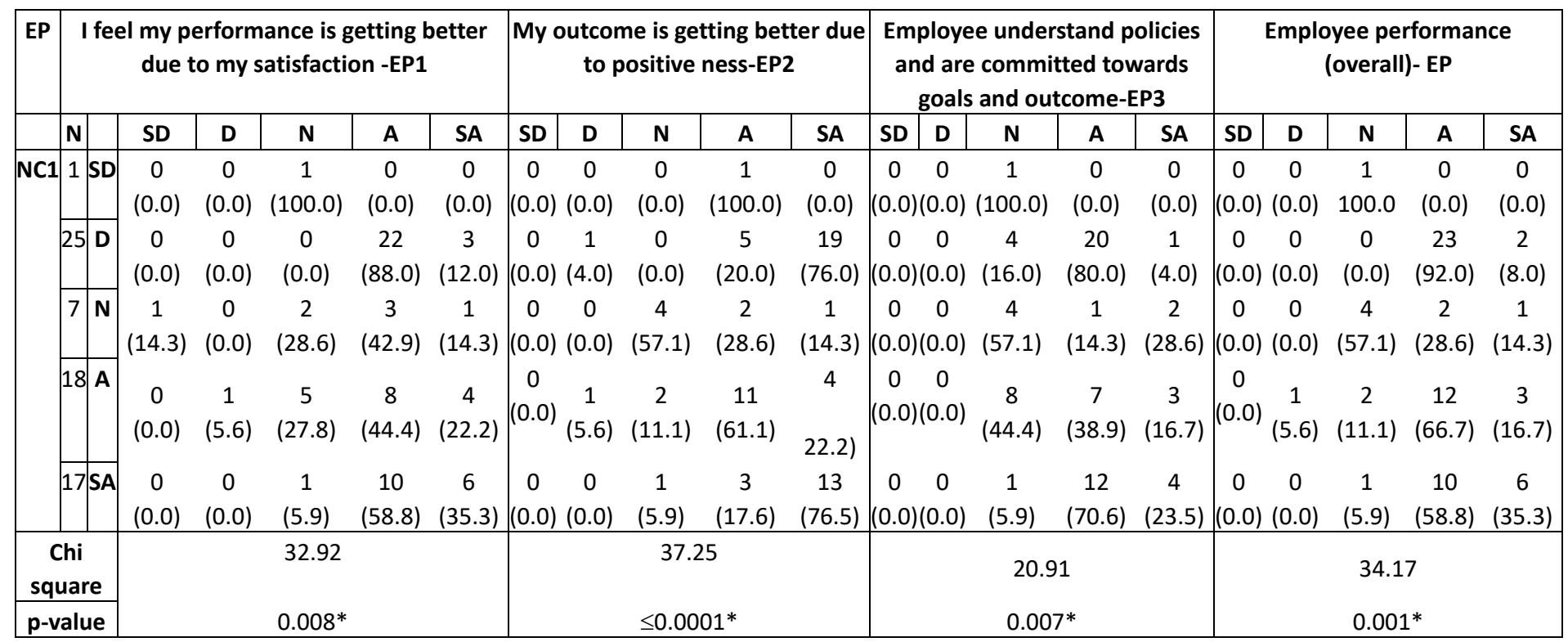

* significant at $\mathrm{p}<0.05$. values within parenthesis are percentage.

SD: strongly agree, D: disagree, N: neutral, A: agree, SA: strongly agree

Table 2 depicts Normative Commitment NC2: "I do not believe that a person must always be loyal to his or her organization." with Employees performance (EP). It is found that $45(66 \%)$ Neutral with NC2. Among them 61.5\% A ,69.2\% SA, 69.2\% A and 69.2\%A with EP1, EP2, EP3 and EP overall, respectively and are statistically significant at $\mathrm{p}<0.05$. 
Table 2. Depicts the Association of Normative Commitment NC2: "I do not believe that a person must always be loyal to his or her organization.” with Employees performance (EP)

\begin{tabular}{|c|c|c|c|c|c|c|c|c|c|c|c|c|c|c|c|c|c|c|c|c|}
\hline \multirow[t]{2}{*}{ EP } & \multicolumn{6}{|c|}{$\begin{array}{l}\text { I feel my performance is getting better } \\
\text { due to my satisfaction -EP1 }\end{array}$} & \multicolumn{5}{|c|}{$\begin{array}{c}\text { My outcome is getting better due } \\
\text { to positive ness-EP2 }\end{array}$} & \multicolumn{4}{|c|}{$\begin{array}{c}\text { Employee understand policies } \\
\text { and are committed towards } \\
\text { goals and outcome-EP3 }\end{array}$} & \multicolumn{5}{|c|}{$\begin{array}{c}\text { Employee performance (overall)- } \\
\text { EP }\end{array}$} \\
\hline & $\mathbf{N}$ & SD & D & $N$ & A & SA & SD & D & $\mathbf{N}$ & A & SA & \begin{tabular}{l|l} 
SD & $D$
\end{tabular} & $\mathbf{N}$ & A & SA & SD & D & $\mathbf{N}$ & A & SA \\
\hline \multirow[t]{5}{*}{ NC2 } & $1 \mathrm{SD}$ & $\begin{array}{c}0 \\
(0.0)\end{array}$ & $\begin{array}{c}0 \\
0 \\
(0.0)\end{array}$ & $\begin{array}{c}0 \\
(0.0)\end{array}$ & $\begin{array}{c}1 \\
(100.0)\end{array}$ & $\begin{array}{c}0 \\
(0.0)\end{array}$ & $\begin{array}{c}0 \\
(0.0)\end{array}$ & $\begin{array}{c}0 \\
(0.0)\end{array}$ & $\begin{array}{c}1 \\
100.0\end{array}$ & $\begin{array}{c}0 \\
(0.0)\end{array}$ & $\begin{array}{c}0 \\
(0.0)\end{array}$ & $\begin{array}{cc}0 & 0 \\
(0.0) & (0.0)\end{array}$ & $\begin{array}{c}1 \\
(100.0)\end{array}$ & $\begin{array}{c}0 \\
0.0)\end{array}$ & $\begin{array}{c}0 \\
(0.0)\end{array}$ & $\begin{array}{c}0 \\
(0.0)\end{array}$ & $\begin{array}{c}0 \\
(0.0)\end{array}$ & $\begin{array}{c}1 \\
(100.0)\end{array}$ & $\begin{array}{c}0 \\
(0.0)\end{array}$ & $\begin{array}{c}0 \\
(0.0)\end{array}$ \\
\hline & $13 \mathrm{D}$ & $\begin{array}{c}1 \\
7.7\end{array}$ & $\begin{array}{c}0 \\
(0.0)\end{array}$ & $\begin{array}{c}3 \\
(23.1)\end{array}$ & $\begin{array}{c}8 \\
(61.5)\end{array}$ & $\begin{array}{c}1 \\
(7.7)\end{array}$ & $\begin{array}{c}0 \\
(0.0)\end{array}$ & $\begin{array}{c}0 \\
(0.0)\end{array}$ & $\begin{array}{c}2 \\
(15.4)\end{array}$ & $\begin{array}{c}2 \\
(15.4)\end{array}$ & $\begin{array}{c}9 \\
(69.2)\end{array}$ & $\begin{array}{cc}0 & 0 \\
(0.0)(0.0)\end{array}$ & $\begin{array}{c}4 \\
(30.8)\end{array}$ & $\begin{array}{c}9 \\
(69.2)\end{array}$ & $\begin{array}{c}0 \\
(0.0)\end{array}$ & $\begin{array}{c}0 \\
(0.0)\end{array}$ & $\begin{array}{c}0 \\
(0.0)\end{array}$ & $\begin{array}{c}3 \\
(23.1)\end{array}$ & $\begin{array}{c}9 \\
(69.2)\end{array}$ & $\begin{array}{c}1 \\
(7.7)\end{array}$ \\
\hline & \begin{tabular}{|l|l|}
$45 \mathbf{N}$ \\
\end{tabular} & $\begin{array}{c}0 \\
(0.0)\end{array}$ & $\begin{array}{c}0 \\
(0.0)\end{array}$ & $\begin{array}{c}5 \\
(11.1)\end{array}$ & $\begin{array}{c}31 \\
(68.9)\end{array}$ & $\begin{array}{c}9 \\
(20.0)\end{array}$ & $\left(\begin{array}{c}0 \\
(0.0)\end{array}\right.$ & $\begin{array}{c}1 \\
(2.2)\end{array}$ & $\begin{array}{c}4 \\
(8.9)\end{array}$ & $\begin{array}{c}15 \\
(33.3)\end{array}$ & $\begin{array}{c}25 \\
(55.6)\end{array}$ & $\begin{array}{cc}0 & 0 \\
(0.0)(0.0)\end{array}$ & $\begin{array}{c}12 \\
(26.7)\end{array}$ & $\begin{array}{c}28 \\
(62.2)\end{array}$ & $\begin{array}{c}5 \\
(11.1)\end{array}$ & $\begin{array}{c}0 \\
(0.0)\end{array}$ & $\begin{array}{c}0 \\
(0.0)\end{array}$ & $\begin{array}{c}4 \\
(8.9)\end{array}$ & $\begin{array}{c}33 \\
(73.3)\end{array}$ & $\begin{array}{c}8 \\
(17.8)\end{array}$ \\
\hline & $\begin{array}{ll}5 & A \\
\end{array}$ & $\begin{array}{c}0 \\
(0.0)\end{array}$ & $\begin{array}{c}1 \\
(20.0)\end{array}$ & $\begin{array}{c}1 \\
(20.0)\end{array}$ & $\begin{array}{c}2 \\
(40.0)\end{array}$ & $\begin{array}{c}1 \\
(20.0)\end{array}$ & $\begin{array}{c}0 \\
(0.0)\end{array}$ & $\begin{array}{c}1 \\
(20.0)\end{array}$ & $\begin{array}{c}0 \\
(0.0)\end{array}$ & $\begin{array}{c}3 \\
(60.0)\end{array}$ & $\begin{array}{c}1 \\
(20.0)\end{array}$ & $\begin{array}{cc}0 & 0 \\
(0.0)(0.0)\end{array}$ & $\begin{array}{c}0 \\
(0.0)\end{array}$ & $\begin{array}{c}2 \\
(40.0)\end{array}$ & $\begin{array}{c}3 \\
(60.0)\end{array}$ & $\begin{array}{c}0 \\
(0.0)\end{array}$ & $\begin{array}{c}1 \\
(20.0)\end{array}$ & $\begin{array}{c}0 \\
(0.0)\end{array}$ & $\begin{array}{c}3 \\
(60.0)\end{array}$ & $\begin{array}{c}1 \\
(20.0)\end{array}$ \\
\hline & $4 \mathrm{SA}$ & $\begin{array}{c}0 \\
(0.0)\end{array}$ & $\begin{array}{c}0 \\
(0.0)\end{array}$ & $\begin{array}{c}0 \\
(0.0)\end{array}$ & $\begin{array}{c}1 \\
(25.0)\end{array}$ & $\begin{array}{c}3 \\
(75.0)\end{array}$ & $\begin{array}{c}0 \\
(0.0)\end{array}$ & $\begin{array}{c}0 \\
(0.0)\end{array}$ & $\begin{array}{c}0 \\
(0.0)\end{array}$ & $\begin{array}{c}2 \\
(50.0)\end{array}$ & $\begin{array}{c}2 \\
(50.0)\end{array}$ & $\begin{array}{cc}0 & 0 \\
(0.0) & (0.0)\end{array}$ & $\begin{array}{c}1 \\
(25.0)\end{array}$ & $\begin{array}{c}1 \\
(25.0)\end{array}$ & $\begin{array}{c}2 \\
(50.0)\end{array}$ & $\begin{array}{c}0 \\
(0.0)\end{array}$ & $\begin{array}{c}0 \\
(0.0)\end{array}$ & $\begin{array}{c}0 \\
(0.0)\end{array}$ & $\begin{array}{c}2 \\
(50.0)\end{array}$ & $\begin{array}{c}2 \\
(50.0)\end{array}$ \\
\hline \multicolumn{2}{|c|}{$\begin{array}{c}\text { Chi } \\
\text { square }\end{array}$} & \multicolumn{5}{|c|}{27.58} & \multicolumn{5}{|c|}{19.95} & \multicolumn{4}{|c|}{18.27} & \multicolumn{5}{|c|}{26.38} \\
\hline \multicolumn{2}{|c|}{$p$-value } & \multicolumn{5}{|c|}{$0.035^{*}$} & \multicolumn{5}{|c|}{0.068} & \multicolumn{4}{|c|}{$0.019 *$} & \multicolumn{5}{|c|}{$0.009 *$} \\
\hline
\end{tabular}

* significant at $\mathrm{p}<0.05$. values within parenthesis are percentage.

SD: strongly agree, D: disagree, N: neutral, A: agree, SA: strongly agree

Table 3 depicts Normative Commitment NC3: "Jumping from organization to organization does not seem at all unethical to me." with Employees performance (EP). It is found that 45(66.1\%) Agree with NC3. Among them $100 \%$ A,50\% A, 0\% A and 50\%A with EP1, EP2, EP3 and EP overall, respectively and are statistically significant at $\mathrm{p}<0.05$.

Table 3. Depicts the Association of Normative Commitment NC3: "Jumping from organization to organization does not seem at all unethical to me." with Employees performance $(\mathrm{EP})$

\begin{tabular}{|c|c|c|c|c|c|c|c|c|c|c|c|c|c|c|c|c|c|c|c|c|}
\hline \multirow[t]{2}{*}{ EP } & \multicolumn{6}{|c|}{$\begin{array}{c}\text { I feel my performance is getting better } \\
\text { due to my satisfaction -EP1 }\end{array}$} & \multicolumn{5}{|c|}{$\begin{array}{c}\text { My outcome is getting better due } \\
\text { to positive ness-EP2 }\end{array}$} & \multicolumn{4}{|c|}{$\begin{array}{c}\text { Employee understand policies } \\
\text { and are committed towards } \\
\text { goals and outcome-EP3 }\end{array}$} & \multicolumn{5}{|c|}{$\begin{array}{l}\text { Employee performance } \\
\text { (overall)- EP }\end{array}$} \\
\hline & $\mathbf{N}$ & SD & D & $\mathbf{N}$ & A & SA & SD & D & $\mathbf{N}$ & A & SA & \begin{tabular}{|l|l|} 
SD & D \\
\end{tabular} & $\mathbf{N}$ & A & SA & SD & D & $\mathbf{N}$ & A & SA \\
\hline \multirow[t]{5}{*}{ NC3 } & 0 SD & $\begin{array}{c}0 \\
(0.0)\end{array}$ & $\begin{array}{c}0 \\
(0.0)\end{array}$ & $\begin{array}{c}0 \\
(0.0)\end{array}$ & $\begin{array}{c}0 \\
(0.0)\end{array}$ & $\begin{array}{c}0 \\
(0.0)\end{array}$ & $\begin{array}{c}0 \\
(0.0)\end{array}$ & $\begin{array}{c}0 \\
(0.0)\end{array}$ & $\begin{array}{c}0 \\
(0.0)\end{array}$ & $\begin{array}{c}0 \\
(0.0)\end{array}$ & $\begin{array}{c}0 \\
(0.0)\end{array}$ & $\begin{array}{cc}0 & 0 \\
(0.0)(0.0)\end{array}$ & $\begin{array}{c}0 \\
(0.0)\end{array}$ & $\begin{array}{c}0 \\
(0.0)\end{array}$ & $\begin{array}{c}0 \\
(0.0)\end{array}$ & $\begin{array}{c}0 \\
(0.0)\end{array}$ & $\begin{array}{c}0 \\
(0.0)\end{array}$ & $\begin{array}{c}0 \\
(0.0)\end{array}$ & $\begin{array}{c}0 \\
(0.0)\end{array}$ & $\begin{array}{c}0 \\
(0.0)\end{array}$ \\
\hline & \begin{tabular}{|l|l|}
2 & $D$ \\
\end{tabular} & $\begin{array}{c}0 \\
(0.0)\end{array}$ & $\begin{array}{c}0 \\
(0.0)\end{array}$ & $\begin{array}{c}0 \\
(0.0)\end{array}$ & $\begin{array}{c}2 \\
100.0\end{array}$ & $\begin{array}{c}0 \\
(0.0)\end{array}$ & $\begin{array}{c}0 \\
0.0)\end{array}$ & $\begin{array}{c}0 \\
(0.0)\end{array}$ & $\begin{array}{c}1 \\
(50.0)\end{array}$ & $\begin{array}{c}1 \\
(50.0)\end{array}$ & $\begin{array}{c}0 \\
(0.0)\end{array}$ & $\begin{array}{cc}0 & 0 \\
(0.0)(0.0)\end{array}$ & $\begin{array}{c}2 \\
(100.0)\end{array}$ & $\begin{array}{c}0 \\
(0.0)\end{array}$ & $\begin{array}{c}0 \\
(0.0)\end{array}$ & $\begin{array}{c}0 \\
(0.0)\end{array}$ & $\begin{array}{c}0 \\
(0.0)\end{array}$ & $\begin{array}{c}1 \\
(50.0)\end{array}$ & $\begin{array}{c}1 \\
(50.0)\end{array}$ & $\begin{array}{c}0 \\
(0.0)\end{array}$ \\
\hline & $14 \mathrm{~N}$ & $\begin{array}{c}1 \\
(7.1)\end{array}$ & $\begin{array}{c}1 \\
(7.1)\end{array}$ & $\begin{array}{c}5 \\
(35.7)\end{array}$ & $\begin{array}{c}4 \\
(28.6)\end{array}$ & $\begin{array}{c}3 \\
(21.4)\end{array}$ & $\begin{array}{c}0 \\
0.0)\end{array}$ & $\begin{array}{c}2 \\
(14.3)\end{array}$ & $\begin{array}{c}4 \\
(28.6)\end{array}$ & $\begin{array}{c}7 \\
(50.0)\end{array}$ & $\begin{array}{c}1 \\
(7.1)\end{array}$ & $\mid \begin{array}{cc}0 & 0 \\
(0.0) & (0.0)\end{array}$ & $\begin{array}{c}5 \\
(35.7)\end{array}$ & $\begin{array}{c}4 \\
(28.6)\end{array}$ & $\begin{array}{c}5 \\
(35.7)\end{array}$ & $\begin{array}{c}0 \\
(0.0)\end{array}$ & $\begin{array}{c}1 \\
(7.1)\end{array}$ & $\begin{array}{c}4 \\
(28.6)\end{array}$ & $\begin{array}{c}7 \\
(50.0)\end{array}$ & $\begin{array}{c}2 \\
(14.3)\end{array}$ \\
\hline & $45 \mathrm{~A}$ & $\begin{array}{c}0 \\
(0.0)\end{array}$ & $\begin{array}{c}0 \\
(0.0)\end{array}$ & $\begin{array}{c}4 \\
(8.9)\end{array}$ & $\begin{array}{c}36 \\
(80.0)\end{array}$ & $\begin{array}{c}5 \\
(11.1)\end{array}$ & $\begin{array}{c}0 \\
(0.0)\end{array}$ & $\begin{array}{c}0 \\
(0.0)\end{array}$ & $\begin{array}{c}2 \\
(4.4)\end{array}$ & $\begin{array}{c}11 \\
(24.4)\end{array}$ & $\begin{array}{c}32 \\
(71.1)\end{array}$ & $\begin{array}{cc}0 & 0 \\
(0.0)(0.0)\end{array}$ & $\begin{array}{c}9 \\
(20.0)\end{array}$ & $\begin{array}{c}34 \\
(75.6)\end{array}$ & $\begin{array}{c}2 \\
(4.4)\end{array}$ & $\begin{array}{c}0 \\
(0.0)\end{array}$ & $\begin{array}{c}0 \\
(0.0)\end{array}$ & $\begin{array}{c}3 \\
(6.7)\end{array}$ & $\begin{array}{c}37 \\
(82.2)\end{array}$ & $\begin{array}{c}5 \\
(11.1)\end{array}$ \\
\hline & $7 \mathrm{SA}$ & $\begin{array}{c}0 \\
(0.0)\end{array}$ & $\begin{array}{c}0 \\
(0.0)\end{array}$ & $\begin{array}{c}0 \\
(0.0)\end{array}$ & $\begin{array}{c}1 \\
14.3\end{array}$ & $\begin{array}{c}6 \\
85.7\end{array}$ & $\begin{array}{c}0 \\
0.0)\end{array}$ & $\begin{array}{c}0 \\
(0.0)\end{array}$ & $\begin{array}{c}0 \\
(0.0)\end{array}$ & $\begin{array}{c}3 \\
(42.9)\end{array}$ & $\begin{array}{c}4 \\
(57.1)\end{array}$ & $\begin{array}{cc}0 & 0 \\
(0.0) & (0.0)\end{array}$ & $\begin{array}{c}2 \\
(28.6)\end{array}$ & $\begin{array}{c}2 \\
(28.6)\end{array}$ & $\begin{array}{c}3 \\
(42.9)\end{array}$ & $\begin{array}{c}0 \\
(0.0)\end{array}$ & $\begin{array}{c}0 \\
(0.0)\end{array}$ & $\begin{array}{c}0 \\
(0.0)\end{array}$ & $\begin{array}{c}2 \\
(28.6)\end{array}$ & $\begin{array}{c}5 \\
(71.4)\end{array}$ \\
\hline \multicolumn{2}{|c|}{$\begin{array}{c}\text { Chi } \\
\text { square }\end{array}$} & \multicolumn{5}{|c|}{39.43} & \multicolumn{5}{|c|}{29.33} & \multicolumn{4}{|c|}{23.33} & \multicolumn{5}{|c|}{28.15} \\
\hline \multicolumn{2}{|c|}{ p-value } & \multicolumn{5}{|c|}{$\leq 0.0001^{*}$} & \multicolumn{5}{|c|}{$0.001 *$} & \multicolumn{4}{|c|}{$0.001^{*}$} & \multicolumn{5}{|c|}{$0.001 *$} \\
\hline
\end{tabular}

* significant at $\mathrm{p}<0.05$. values within parenthesis are percentage. 


\section{Macrothink}

International Journal of Human Resource Studies

ISSN 2162-3058

2021, Vol. 11, No. 3

SD: strongly agree, D: disagree, N: neutral, A: agree, SA: strongly agree

Table 4 depicts Normative Commitment NC4: "One of the major reasons I continue to work in this organization is that I believe loyalty is important and therefore feel a sense of moral obligation to remain" with Employees performance (EP). It is found that 34(50\%) Agree with NC4. Among them 25\% SA,75\% A, 50\% A and 50\%A with EP1, EP2, EP3 and EP overall, respectively and are statistically significant at $\mathrm{p}<0.05$.

Table 4. Depicts the Association of Normative Commitment NC4: "One of the major reasons I continue to work in this organization is that I believe loyalty is important and therefore feel a sense of moral obligation to remain" with Employees performance (EP)

\begin{tabular}{|c|c|c|c|c|c|c|c|c|c|c|c|c|c|c|c|c|c|c|c|c|c|}
\hline EP & I fee & $\begin{array}{l}\text { my pe } \\
\text { due } t\end{array}$ & $\begin{array}{l}\text { erform } \\
\text { o my s }\end{array}$ & $\begin{array}{l}\text { ance is } \\
\text { atisfact }\end{array}$ & $\begin{array}{l}\text { getting } \\
\text { ion -EP }\end{array}$ & better & & $\begin{array}{l}\text { youtc } \\
\text { due to }\end{array}$ & positive is & $\begin{array}{l}\text { jetting } \\
\text { e ness- }\end{array}$ & $\begin{array}{l}\text { better } \\
\text { EP2 }\end{array}$ & & $\begin{array}{l}\text { ploye } \\
\text { d are } \\
\text { goals }\end{array}$ & $\begin{array}{l}\text { ee unde } \\
\text { e comm } \\
\text { s and ou }\end{array}$ & $\begin{array}{l}\text { arstand } p \\
\text { itted tou } \\
\text { utcome-E }\end{array}$ & $\begin{array}{l}\text { olicies } \\
\text { Nards } \\
\text { EP3 }\end{array}$ & & Emplc & $\begin{array}{l}\text { Dyee pe } \\
\text { (overall }\end{array}$ & $\begin{array}{l}\text { formar } \\
\text { EP }\end{array}$ & \\
\hline & $\mathbf{N}$ & SD & D & $\mathbf{N}$ & A & SA & SD & D & $\mathbf{N}$ & A & SA & SD & D & $\mathbf{N}$ & A & SA & SD & D & $\mathbf{N}$ & A & SA \\
\hline NC4 & $2 \mathrm{SD}$ & $\begin{array}{c}0 \\
(0.0)\end{array}$ & $\begin{array}{c}0 \\
(0.0)\end{array}$ & $\begin{array}{c}0 \\
(0.0)\end{array}$ & $\begin{array}{c}0 \\
(0.0)\end{array}$ & $\begin{array}{c}2 \\
100.0\end{array}$ & $\begin{array}{c}0 \\
(0.0)\end{array}$ & $\begin{array}{c}0 \\
(0.0)\end{array}$ & $\begin{array}{c}0 \\
(0.0)\end{array}$ & $\begin{array}{c}0 \\
(0.0)\end{array}$ & $\begin{array}{c}2 \\
100.0\end{array}$ & $\begin{array}{c}0 \\
(0.0)\end{array}$ & $\begin{array}{c}0 \\
(0.0)\end{array}$ & $\begin{array}{c}0 \\
(0.0)\end{array}$ & $\begin{array}{c}2 \\
(100.0)\end{array}$ & $\begin{array}{c}0 \\
(0.0)\end{array}$ & $\begin{array}{c}0 \\
(0.0)\end{array}$ & $\begin{array}{c}0 \\
(0.0)\end{array}$ & $\begin{array}{c}0 \\
(0.0)\end{array}$ & $\begin{array}{c}0 \\
(0.0)\end{array}$ & $\begin{array}{c}2 \\
100.0\end{array}$ \\
\hline & 4 D & $\begin{array}{c}1 \\
(25.0)\end{array}$ & $\begin{array}{c}0 \\
(0.0)\end{array}$ & $\begin{array}{c}2 \\
(50.0)\end{array}$ & $\begin{array}{c}0 \\
(0.0)\end{array}$ & $\begin{array}{c}1 \\
(25.0)\end{array}$ & $\begin{array}{c}0 \\
(0.0)\end{array}$ & $\begin{array}{c}0 \\
(0.0)\end{array}$ & $\begin{array}{c}1 \\
(25.0)\end{array}$ & $\begin{array}{c}3 \\
(75.0)\end{array}$ & $\begin{array}{c}0 \\
(0.0)\end{array}$ & $\begin{array}{c}0 \\
(0.0)\end{array}$ & $\begin{array}{c}0 \\
(0.0)\end{array}$ & $\begin{array}{c}2 \\
(50.0)\end{array}$ & $\begin{array}{c}2 \\
(50.0)\end{array}$ & $\begin{array}{c}0 \\
(0.0)\end{array}$ & $\begin{array}{c}0 \\
(0.0)\end{array}$ & $\begin{array}{c}0 \\
(0.0)\end{array}$ & $\begin{array}{c}2 \\
(50.0)\end{array}$ & $\begin{array}{c}2 \\
(50.0)\end{array}$ & $\begin{array}{c}0 \\
(0.0)\end{array}$ \\
\hline & $24 \mathrm{~N}$ & $\begin{array}{c}0 \\
(0.0)\end{array}$ & $\begin{array}{c}0 \\
(0.0)\end{array}$ & $\begin{array}{c}4 \\
(16.7)\end{array}$ & $\begin{array}{c}15 \\
(62.5)\end{array}$ & $\begin{array}{c}5 \\
(20.8)\end{array}$ & $\begin{array}{c}0 \\
(0.0)\end{array}$ & $\begin{array}{c}0 \\
(0.0)\end{array}$ & $\begin{array}{c}3 \\
(12.5)\end{array}$ & $\begin{array}{c}14 \\
(58.3)\end{array}$ & $\begin{array}{c}7 \\
(29.2)\end{array}$ & $\begin{array}{c}0 \\
(0.0)\end{array}$ & $\begin{array}{c}0 \\
(0.0)\end{array}$ & $\begin{array}{c}12 \\
(50.0)\end{array}$ & $\begin{array}{c}8 \\
(33.3)\end{array}$ & $\begin{array}{c}4 \\
(16.7)\end{array}$ & $\begin{array}{c}0 \\
(0.0)\end{array}$ & $\begin{array}{c}0 \\
(0.0)\end{array}$ & $\begin{array}{c}3 \\
(12.5)\end{array}$ & $\begin{array}{c}15 \\
(62.5)\end{array}$ & $\begin{array}{c}6 \\
(25.0)\end{array}$ \\
\hline & $34 \mathrm{~A}$ & $\begin{array}{c}0 \\
(0.0)\end{array}$ & $\begin{array}{c}1 \\
(2.9)\end{array}$ & $\begin{array}{c}3 \\
(8.8)\end{array}$ & $\begin{array}{c}27 \\
(79.4)\end{array}$ & $\begin{array}{c}3 \\
(8.8)\end{array}$ & $\begin{array}{c}0 \\
(0.0)\end{array}$ & $\begin{array}{c}2 \\
(5.9)\end{array}$ & $\begin{array}{c}3 \\
(8.8)\end{array}$ & $\begin{array}{c}3 \\
(8.8)\end{array}$ & $\begin{array}{c}26 \\
(76.5)\end{array}$ & $\begin{array}{c}0 \\
(0.0)\end{array}$ & $\begin{array}{c}0 \\
(0.0)\end{array}$ & $\begin{array}{c}3 \\
(8.8)\end{array}$ & $\begin{array}{c}27 \\
(79.4)\end{array}$ & $\begin{array}{c}4 \\
(11.8)\end{array}$ & $\begin{array}{c}0 \\
(0.0)\end{array}$ & $\begin{array}{c}1 \\
(2.9)\end{array}$ & $\begin{array}{c}3 \\
(8.8)\end{array}$ & $\begin{array}{c}28 \\
(82.4)\end{array}$ & $\begin{array}{c}2 \\
(5.9)\end{array}$ \\
\hline & 4 SA & $\begin{array}{c}0 \\
(0.0)\end{array}$ & $\begin{array}{c}0 \\
(0.0)\end{array}$ & $\begin{array}{c}0 \\
(0.0)\end{array}$ & $\begin{array}{c}1 \\
(25.0)\end{array}$ & $\begin{array}{c}3 \\
(75.0)\end{array}$ & $\begin{array}{c}0 \\
(0.0)\end{array}$ & $\begin{array}{c}0 \\
(0.0)\end{array}$ & $\begin{array}{c}0 \\
(0.0)\end{array}$ & $\begin{array}{c}2 \\
(50.0)\end{array}$ & $\begin{array}{c}2 \\
(50.0)\end{array}$ & $\begin{array}{c}0 \\
(0.0)\end{array}$ & $\begin{array}{c}0 \\
(0.0)\end{array}$ & $\begin{array}{c}1 \\
(25.0)\end{array}$ & $\begin{array}{c}1 \\
(25.0)\end{array}$ & $\begin{array}{c}2 \\
(50.0)\end{array}$ & $\begin{array}{c}0 \\
(0.0)\end{array}$ & $\begin{array}{c}0 \\
(0.0)\end{array}$ & $\begin{array}{c}0 \\
(0.0)\end{array}$ & $\begin{array}{c}2 \\
(50.0)\end{array}$ & $\begin{array}{c}2 \\
(50.0)\end{array}$ \\
\hline & & & & 42.92 & & & & & 26.5 & & & & & 21. & & & & & 23.9 & & \\
\hline & lue & & & .000 & & & & & 0.0 & & & & & & & & & & 0. & & \\
\hline
\end{tabular}

* significant at $\mathrm{p}<0.05$. values within parenthesis are percentage.

SD:strongly agree, D:disagree, N:neutral, A:agree, SA:strongly agree

Table 5 depicts of Normative Commitment NC5: "If I got another offer for a better job, elsewhere I would not feel it was right to leave my organization" with Employees performance (EP). It is found that 36(52.9\%) Agree with NC5. Among them $100 \%$ were neutral with EP1, EP2, EP3 and EP overall, respectively and are statistically significant at $\mathrm{p}<0.05$. 
Table 5. Depicts the Association of Normative Commitment NC5: "If I got another offer for a better job, elsewhere I would not feel it was right to leave my organization" with Employees performance (EP)

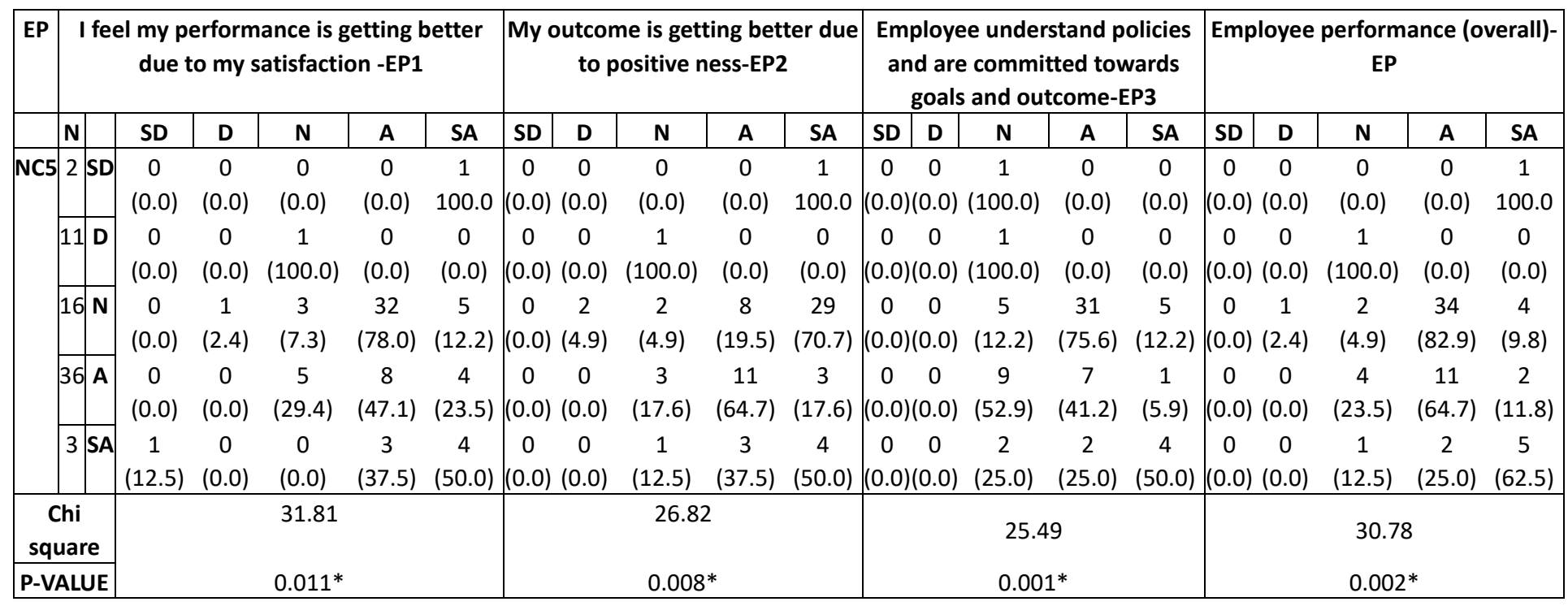

* significant at $\mathrm{p}<0.05$. values within parenthesis are percentage.

SD: strongly agree, D: disagree, N: neutral, A: agree, SA: strongly agree

Table 6 depicts Normative Commitment NC6: "I was taught to believe in the value of remaining loyal to one organization" with Employees performance (EP). It is found that 36(62\%) Agree with NC6. Among them 100\% Agreed with EP1, EP2, EP3 and EP overall, respectively and are statistically significant at $\mathrm{p}<0.05$.

Table 6. Depicts the Association of Normative Commitment NC6: "I was taught to believe in the value of remaining loyal to one organization" with Employees performance (EP)

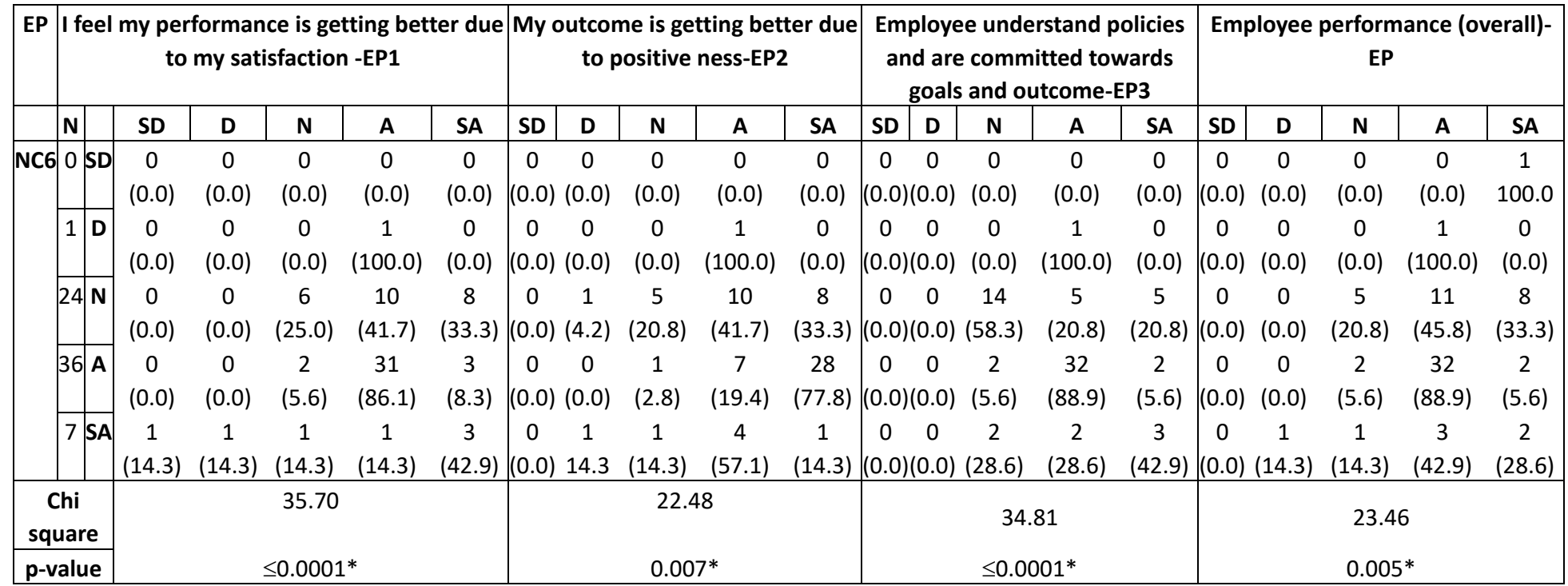

* significant at $\mathrm{p}<0.05$. values within parenthesis are percentage.

SD: strongly agree, D: disagree, N: neutral, A: agree, SA: strongly agree 


\section{Macrothink}

International Journal of Human Resource Studies ISSN 2162-3058 2021, Vol. 11, No. 3

Table 7 depicts Normative Commitment NC7: "Things were better in the days when people stayed in one organization for most of their careers" with Employees performance (EP). It is found only that $9(13 \%)$ Agree with NC7. Among them 33.3\%A with EP1, EP2, EP3 and 33.3\% SA with EP overall, respectively and are statistically significant at $\mathrm{p}<0.05$.

Table 7. Depicts the Association of Normative Commitment NC7: "Things were better in the days when people stayed in one organization for most of their careers" with Employees performance $(\mathrm{EP})$

\begin{tabular}{|c|c|c|c|c|c|c|c|c|c|c|c|c|c|c|c|c|c|c|c|c|c|}
\hline \multirow[t]{2}{*}{ EP } & \multicolumn{6}{|c|}{$\begin{array}{l}\text { I feel my performance is getting better } \\
\text { due to my satisfaction -EP1 }\end{array}$} & \multicolumn{5}{|c|}{$\begin{array}{c}\text { My outcome is getting better due } \\
\text { to positive ness-EP2 }\end{array}$} & \multicolumn{5}{|c|}{$\begin{array}{c}\text { Employee understand policies and } \\
\text { are committed towards goals and } \\
\text { outcome-EP3 }\end{array}$} & \multicolumn{5}{|c|}{$\begin{array}{l}\text { Employee performance (overall)- } \\
\text { EP }\end{array}$} \\
\hline & & SD & D & $\mathbf{N}$ & A & SA & SD & D & $\mathbf{N}$ & A & SA & SD & D & $\mathbf{N}$ & A & SA & SD & D & $\mathbf{N}$ & A & SA \\
\hline \multirow{4}{*}{ NC7 } & $0 \mathrm{SD}$ & 0 & 0 & 0 & 0 & 0 & 0 & 0 & 0 & 0 & 0 & 0 & 0 & 0 & 0 & 0 & 0 & 0 & 0 & 0 & 0 \\
\hline & & $(0.0)$ & $(0.0)$ & $(0.0)$ & $(0.0)$ & $(0.0)$ & $(0.0)$ & $(0.0)$ & $(0.0)$ & $(0.0)$ & $(0.0)$ & $(0.0)$ & $(0.0)$ & $(0.0)$ & $(0.0)$ & $(0.0)$ & $(0.0)$ & $(0.0)$ & (0.0) & (0.0) & (0.0) \\
\hline & \begin{tabular}{l|l|}
3 & $D$ \\
\end{tabular} & 0 & 1 & 1 & 0 & 1 & 0 & 1 & 0 & 1 & 1 & 0 & 0 & 1 & 1 & 1 & 0 & 1 & 1 & 0 & 1 \\
\hline & & $(0.0)$ & (33.3) & (33.3) & $(0.0)$ & (33.3) & $(0.0)$ & (33.3) & $(0.0)$ & (33.3) & (33.3) & $(0.0)$ & $(0.0)$ & (33.3) & (33.3) & (33.3) & $(0.0)$ & (33.3) & (33.3) & $(0.0)$ & (33.3) \\
\hline & $53 \mathrm{~N}$ & 0 & 0 & 7 & 39 & 7 & 0 & 0 & 4 & 16 & 33 & 0 & 0 & 11 & 35 & 7 & 0 & 0 & 4 & 42 & 7 \\
\hline & & $(0.0)$ & $(0.0)$ & (13.2) & (73.6) & (13.2) & $(0.0)$ & $(0.0)$ & $(7.5)$ & (30.2) & (62.3) & $(0.0)$ & $(0.0)$ & (20.8) & (66.0) & (13.2) & (0.0) & $(0.0)$ & (7.5) & (79.2) & (13.2) \\
\hline & \begin{tabular}{|l|l|}
9 & A \\
\end{tabular} & 0 & 0 & 1 & 3 & 5 & 0 & 1 & 2 & 3 & 3 & 0 & 0 & 3 & 4 & 2 & 0 & 0 & 2 & 3 & 4 \\
\hline & & $(0.0)$ & $(0.0)$ & (11.1) & (33.3) & (55.6) & $(0.0)$ & (11.1) & (22.2) & (33.3) & (33.3) & $(0.0)$ & $(0.0)$ & (33.3) & (44.4) & $(22.2)$ & $(0.0)$ & $(0.0)$ & $(22.2)$ & (33.3) & $(44.4)$ \\
\hline & $3 \mathrm{SA}$ & 1 & 0 & 0 & 1 & 1 & 0 & 0 & 1 & 2 & 0 & 0 & 0 & 3 & 0 & 0 & 0 & 0 & 1 & 2 & 0 \\
\hline & & (33.3) & $(0.0)$ & (0.0) & (33.3) & 33.3 & $(0.0)$ & $(0.0)$ & 33.3 & (66.7) & $(0.0)$ & $(0.0)$ & $(0.0)$ & 100.0 & $(0.0)$ & $(0.0)$ & $(0.0)$ & $(0.0)$ & 33.3 & (66.7) & $(0.0)$ \\
\hline \multicolumn{2}{|c|}{$\begin{array}{c}\text { Chi } \\
\text { square }\end{array}$} & \multicolumn{5}{|c|}{56.38} & \multicolumn{5}{|c|}{20.98} & \multicolumn{5}{|c|}{11.45} & \multicolumn{5}{|c|}{35.40} \\
\hline \multicolumn{2}{|c|}{$p$-value } & \multicolumn{5}{|c|}{$\leq 0.0001^{*}$} & \multicolumn{5}{|c|}{$0.013 *$} & \multicolumn{5}{|c|}{0.075} & \multicolumn{5}{|c|}{$\leq 0.0001^{*}$} \\
\hline
\end{tabular}

* significant at $\mathrm{p}<0.05$. values within parenthesis are percentage.

SD: strongly agree, D: disagree, N: neutral, A: agree, SA: strongly agree

Table 8 depicts Normative Commitment NC8: "I do not think that to be a 'company man' or 'company woman' is sensible anymore" with Employees performance (EP). It is found that 32(47\%) Agree with NC8. Among them 66.7\% A ,33.3\% SA, 66.7\% SA and 33.3\%A with $\mathrm{EP} 1, \mathrm{EP} 2, \mathrm{EP} 3$ and EP overall, respectively and are statistically significant at $\mathrm{p}<0.05$. 


\section{Macrothink Institute ${ }^{\text {th }}$}

Table 8. Depicts the Association of Normative Commitment NC8: "I do not think that to be a 'company man' or 'company woman' is sensible anymore" with Employees performance (EP)

\begin{tabular}{|c|c|c|c|c|c|c|c|c|c|c|c|c|c|c|c|c|c|c|c|c|c|}
\hline \multirow{2}{*}{\begin{tabular}{|l|} 
EP \\
\\
\end{tabular}} & \multicolumn{6}{|c|}{$\begin{array}{l}\text { I feel my performance is getting better } \\
\text { due to my satisfaction -EP1 }\end{array}$} & \multicolumn{5}{|c|}{$\begin{array}{l}\text { My outcome is getting better due } \\
\text { to positive ness-EP2 }\end{array}$} & \multicolumn{5}{|c|}{$\begin{array}{c}\text { Employee understand policies and } \\
\text { are committed towards goals and } \\
\text { outcome-EP3 }\end{array}$} & \multicolumn{5}{|c|}{ Employee performance (overall)- EP } \\
\hline & $\mathbf{N}$ & SD & D & $\mathbf{N}$ & A & SA & SD & D & $\mathbf{N}$ & A & SA & SD & $\mathbf{D}$ & $\mathbf{N}$ & A & SA & SD & D & $\mathbf{N}$ & A & SA \\
\hline \multirow[t]{10}{*}{ NC8 } & \begin{tabular}{|l|l|}
2 & $S D$ \\
\end{tabular} & 0 & 0 & 0 & 0 & 2 & 0 & 1 & 0 & 1 & 0 & 0 & 0 & 0 & 0 & 2 & 0 & 0 & 0 & 0 & 2 \\
\hline & & $(0.0)$ & $(0.0)$ & $(0.0)$ & $(0.0)$ & $(100.0)$ & $(0.0)$ & $(50.0)$ & 0.0 & $(50.0)$ & 0.0 & $(0.0)$ & $(0.0)$ & $(0.0)$ & $(0.0)$ & $(100.0)$ & $(0.0)$ & $(0.0)$ & $(0.0)$ & $(0.0)$ & (100.0) \\
\hline & \begin{tabular}{|l|l|}
3 & $D$ \\
\end{tabular} & 0 & 1 & 0 & 2 & 0 & 0 & 1 & 0 & 1 & 1 & 0 & 0 & 1 & 0 & 2 & 0 & 1 & 0 & 1 & 1 \\
\hline & & $(0.0)$ & (33.3) & $(0.0)$ & $(66.7)$ & $(0.0)$ & $(0.0)$ & (33.3) & 0.0 & (33.3) & (33.3) & $(0.0)$ & $(0.0)$ & (33.3) & $(0.0)$ & (66.7) & $(0.0)$ & (33.3) & $(0.0)$ & (33.3) & (33.3) \\
\hline & \begin{tabular}{|l|l|}
$22 \mathrm{~N}$ \\
\end{tabular} & 0 & 0 & 7 & 9 & 6 & 0 & 0 & 4 & 11 & 7 & 0 & 0 & 12 & 6 & 4 & 0 & 0 & 5 & 11 & 6 \\
\hline & & $(0.0)$ & $(0.0)$ & (31.8) & (40.9) & (27.3) & $(0.0)$ & $(0.0)$ & (18.2) & $(50.0)$ & (31.8) & $(0.0)$ & $(0.0)$ & (54.5) & (27.3) & $(18.2)$ & $(0.0)$ & $(0.0)$ & (22.7) & $(50.0)$ & (27.3) \\
\hline & \begin{tabular}{|l|l|}
32 & $\mathrm{~A}$ \\
\end{tabular} & 1 & 0 & 2 & 28 & 1 & 0 & 0 & 3 & 2 & 27 & 0 & 0 & 3 & 27 & 2 & 0 & 0 & 3 & 28 & 1 \\
\hline & & (3.1) & $(0.0)$ & (6.3) & (87.5) & (3.1) & $(0.0)$ & $(0.0)$ & $(9.4)$ & (6.3) & (84.4) & $(0.0)$ & $(0.0)$ & $(9.4)$ & (84.4) & (6.3) & $(0.0)$ & 0.0 & (9.4) & (87.5) & (3.1) \\
\hline & \begin{tabular}{|l|l|}
9 & SA \\
\end{tabular} & 0 & 0 & 0 & 4 & 5 & 0 & 0 & 0 & 7 & 2 & 0 & 0 & 2 & 7 & 0 & 0 & 0 & 0 & 7 & 2 \\
\hline & & $(0.0)$ & $(0.0)$ & $(0.0)$ & $(44.4)$ & (55.6) & $(0.0)$ & $(0.0)$ & $(0.0)$ & (77.8) & (22.2) & $(0.0)$ & $(0.0)$ & $(22.2)$ & (77.8) & $(0.0)$ & $(0.0)$ & $(0.0)$ & $(0.0)$ & $(77.8)$ & $(22.2)$ \\
\hline \multicolumn{2}{|c|}{$\begin{array}{c}\text { Chi } \\
\text { square }\end{array}$} & \multicolumn{5}{|c|}{55.35} & \multirow{2}{*}{\multicolumn{5}{|c|}{54.31}} & \multirow{2}{*}{\multicolumn{5}{|c|}{39.95}} & \multicolumn{5}{|c|}{43.67} \\
\hline \multicolumn{2}{|c|}{$p$-value } & \multicolumn{5}{|c|}{$\leq 0.0001 *$} & & & & & & & & & & & \multicolumn{5}{|c|}{$\leq 0.0001 *$} \\
\hline
\end{tabular}

* significant at $\mathrm{p}<0.05$. values within parenthesis are percentage.

SD: strongly agree, D: disagree, N: neutral, A: agree, SA: strongly agree

Table-9 shows Association of overall Normative Commitment (NC) with Employees Job Performance (EP). It is found that 34(44\%) agree with that Normative Commitment (NC) is closely associated with Employees job Performance. From analysis it is clear that $52.2 \%$ A, 26.1\% SA, 30.4\% A and 60.9\% A with EP1, EP2, EP3 and EP overall, respectively and are statistically significant at $\mathrm{p}<0.05$.

Table 9. Depicts the Association of overall Normative Commitment (NC) with Employees Job Performance (EP)

\begin{tabular}{|c|c|c|c|c|c|c|c|c|c|c|c|c|c|c|c|c|c|c|c|c|c|}
\hline \multirow{2}{*}{\begin{tabular}{l|l|} 
EP \\
\end{tabular}} & \multicolumn{6}{|c|}{$\begin{array}{l}\text { I feel my performance is getting better } \\
\text { due to my satisfaction -EP1 }\end{array}$} & \multicolumn{5}{|c|}{$\begin{array}{l}\text { My outcome is getting better due } \\
\text { to positive ness-EP2 }\end{array}$} & \multicolumn{5}{|c|}{$\begin{array}{c}\text { Employee understand policies and } \\
\text { are committed towards goals and } \\
\text { outcome-EP3 }\end{array}$} & \multicolumn{5}{|c|}{$\begin{array}{c}\text { Employee performance (overall)- } \\
\text { EP }\end{array}$} \\
\hline & $\mathbf{N}$ & SD & D & $\mathbf{N}$ & A & SA & SD & D & $\mathbf{N}$ & A & SA & SD & D & $\mathbf{N}$ & A & SA & SD & D & $\mathbf{N}$ & A & SA \\
\hline \multirow[t]{10}{*}{ Overal } & 10 SD & 0 & 0 & 0 & 0 & 0 & 0 & 0 & 0 & 0 & 0 & 0 & 0 & 0 & 0 & 0 & 0 & 0 & 0 & 0 & 0 \\
\hline & & $(0.0)$ & $(0.0)$ & $(0.0)$ & $(0.0)$ & $(0.0)$ & $(0.0)$ & $(0.0)$ & $(0.0)$ & $(0.0)$ & $(0.0)$ & $(0.0)$ & $(0.0)$ & $(0.0)$ & $(0.0)$ & $(0.0)$ & $(0.0)$ & $(0.0)$ & $(0.0)$ & $(0.0)$ & (0.0) \\
\hline & 1 D & 0 & 0 & 1 & 0 & 0 & 0 & 0 & 0 & 1 & 0 & 0 & 0 & 1 & 0 & 0 & 0 & 0 & 1 & 0 & 0 \\
\hline & & $(0.0)$ & $(0.0)$ & $(100.0)$ & $(0.0)$ & $(0.0)$ & $(0.0)$ & $(0.0)$ & $(0.0)$ & (100.0) & $(0.0)$ & $(0.0)$ & $(0.0)$ & (100.0) & $(0.0)$ & $(0.0)$ & $(0.0)$ & $(0.0)$ & (100.0) & $(0.0)$ & (0.0) \\
\hline & \begin{tabular}{|l|l|}
23 & $N$ \\
\end{tabular} & 0 & 0 & 6 & 12 & 5 & 0 & 1 & 4 & 12 & 6 & 0 & 0 & 13 & 7 & 3 & 0 & 0 & 4 & 14 & 5 \\
\hline & & $(0.0)$ & $(0.0)$ & (26.1) & (52.2) & (21.7) & $(0.0)$ & (4.3) & (17.4) & $(52.2)$ & (26.1) & $(0.0)$ & $(0.0)$ & (56.5) & (30.4) & (13.0) & $(0.0)$ & $(0.0)$ & (17.4) & 60.9 & $(21.7)$ \\
\hline & 34 A & 0 & 1 & 2 & 30 & 1 & 0 & 1 & 2 & 6 & 25 & 0 & 0 & 2 & 30 & 2 & 0 & 1 & 2 & 31 & 0 \\
\hline & & $(0.0)$ & (2.9) & (5.9) & (88.2) & $(2.9)$ & $(0.0)$ & (2.9) & (5.9) & $(17.6)$ & (73.5) & $(0.0)$ & $(0.0)$ & (5.9) & (88.2) & (5.9) & $(0.0)$ & (2.9) & (5.9) & 91.2 & $(0.0)$ \\
\hline & $10 \mathrm{SA}$ & 1 & 0 & 0 & 1 & 8 & 0 & 0 & 1 & 3 & 6 & 0 & 0 & 2 & 3 & 5 & 0 & 0 & 1 & 2 & 7 \\
\hline & & $(10.0)$ & $(0.0)$ & $(0.0)$ & $(10.0)$ & $(80.0)$ & $(0.0)$ & $(0.0)$ & $(10.0)$ & $(30.0)$ & $(60.0)$ & $(0.0)$ & $(0.0)$ & $(20.0)$ & $(30.0)$ & $(50.0)$ & $(0.0)$ & $(0.0)$ & $(10.0)$ & $(20.0)$ & $(70.0)$ \\
\hline \multirow{2}{*}{\multicolumn{2}{|c|}{ Chi square }} & \multirow{2}{*}{\multicolumn{5}{|c|}{$\begin{aligned} & 49.49 \\
\leq & 0.0001^{*}\end{aligned}$}} & \multirow{2}{*}{\multicolumn{5}{|c|}{$\begin{array}{l}15.12 \\
0.088\end{array}$}} & \multirow{2}{*}{\multicolumn{5}{|c|}{$\begin{array}{c}36.10 \\
\leq 0.0 .0001 *\end{array}$}} & \multirow{2}{*}{\multicolumn{5}{|c|}{$\begin{array}{c}37.99 \\
\leq 0.0001 *\end{array}$}} \\
\hline p-val & & & & & & & & & & & & & & & & & & & & & \\
\hline
\end{tabular}


* significant at $\mathrm{p}<0.05$. values within parenthesis are percentage.

SD: strongly agree, D: disagree, N: neutral, A: agree, SA: strongly agree

From the above analysis it is clear that there is a close association between Normative Commitment (NC) and employees performance in sample study organization.

\section{Conclusion}

The main objective of this research study was to investigate the association between Normative Commitment (Sense of Obligation to Stay) and employees' performance in FENDA Communication and IT. From analysis it is clear that there is significant positive association between Normative Commitment and an employee's performance. Only 14\% respondents agreed with $\mathrm{NC1}$ statement that shows employees are satisfy with the organization, they will not leave the organization and in sample organization the level of satisfaction is high among the employees. For NC2 statement, 66\% employees were neutral and $7 \%$ agreed that employees should be loyal towards organization. It is also clear from analysis $66 \%$ of respondent agreed with NC3 that indicates moving from one company to other company is not unethical nowadays it is considered as opportunity. About $50 \%$ of respondents agreed with NC4 statement because they feel a sense of moral obligation to stay with existing company. For NC5 and NC6 statement, 60\% respondents agreed that if they will get better offer elsewhere, they will not leave the current company and will remain loyal to their company. Only $11 \%$ of respondents agreed with NC7 statement that things were better in the days when people stayed in one organization for most of their careers. About $50 \%$ respondents agreed with NC8 that to be a 'company man' or 'company woman' is not sensible anymore". Most of the respondents agreed that there is close association between Normative Commitment and an employee's performance in sample study organization.

\section{Limitations and Future Directions}

The survey was conducted with small size of sample of 68 respondents, and therefore results cannot be generalized. Future studies should consider large sample size. This research included only IT company for research purpose, but future research should consider other service sectors like education, tourism, hospital and insurance sector.

\section{Acknowledgement}

Author would like to acknowledge Deanship of Graduate and Research Studies of Dar Al Uloom University (DAU), Saudi Arabia Riyadh for financial support. Author is exceptionally indebted to Professor Abdulrahman Alsultan, Dean of the College of Business for his motivation, enthusiasm and supportive for my career advancement.

\section{References}

Allen, N. J., \& Meyer, J. P, (1990). The measurement and antecedents of affective, continuance and normative commitment to the organization. Journal of occupational psychology, 63(1), 1-18. https://doi.org/10.1111/j.2044-8325.1990.tb00506.x

Bingöl, D. (2003). İnsan Kaynakları Yönetimi, Beta Yayınları, İstanbul. 


\section{Mll Macrothink}

International Journal of Human Resource Studies ISSN 2162-3058 2021, Vol. 11, No. 3

Chen, J., Silverthorne, C., \& Hung, J. (2006). Organization communication, job stress, organizational commitment, and job performance of accounting professionals in Taiwan and America. Leadership \& Organization Development Journal, 27(4), 242-249. https://doi.org/10.1108/01437730610666000

Dharma, A. (1991). Manajemen Prestasi. Jakarta: Rajawali Press.

Dixit, V., \& Bhati, M. (2012). A Study about Employee Commitment and its impact on Sustained Productivity in Indian Auto-Component Industry. European Journal of Business and Social Sciences, 1(6), $34-51$.

Harter, J. K., Schmidt, F. L., \& Hayes, T. L. (2002). Business Unit Level Relationship between Employee Satisfaction, Employee Engagement, and Business Outcomes: A Meta-Analysis. Journal of Applied Psychology, 87(2), 268- 279. https://doi.org/10.1037/0021-9010.87.2.268

Herscovitch, L., \& Meyer, J. P. (2002). Commitment to organizational change: Extension of a three-component model. Journal of Applied Psychology, 87, 474-487. https://doi.org/10.1037/0021-9010.87.3.474

Iraz, R., \& Akgün, Ö. (2011). Örgütsel bağlılığın çalışan performansı üzerine etkilerini ölçmeye yönelik bir çalışma. Sosyal Bilimler Meslek Yüksekokulu Dergisi, 14(1-2), 225-250.

Meyer, J. P., Stanley, D. J., Herscovitch, L., \& Topolnytsky, L. (2002). Affective, Continuance and Normative Commitment to the Organization: a Meta-analysis of Antecedents, Correlates and Consequences. Journal of Vocational Behavior, 61, 21-52. https://doi.org/10.1006/jvbe.2001.1842

Meyer, J. P., \& Allen, N. J. (1990). The measurement and antecedents of affective, continuance and normative commitment to the organization. Journal of occupational psychology, 63(1), 1-18. https://doi.org/10.1111/j.2044-8325.1990.tb00506.x

Meyer, J. P., \& Allen, N. J. (1991). A three-component conceptualization of organizational commitment. Human Resource Management Review, 1, 64-89. https://doi.org/10.1016/1053-4822(91)90011-Z

Meyer, J. P., \& Allen, N. J. (1997). Commitment in the workplace: Theory, research, and application. Thousand Oaks, CA: Sage.

Meyer, J. P., \& Herscovitch, L. (2001). Commitment in the workplace: Toward the general model. Human Resource Management Review, 11, 299-326.

Özutku, H. (2008). Örgüte duygusal, devamlılık ve normative bağl1lık ile iş performansı arasındaki ilişkinin incelenmesi. İstanbul Üniversitesi İşletme Fakültesi Dergisi, 37(2), 79-97.

Prawirosentoso, S. (2000). Manajemen operasi : analisis dan studi kasus Ediga: Ketigu Bumi Aksara, Jakarta.

Qaisar, M. U., Rehman, M. S., \& Suffyan, M. (2012). Exploring Effects of Organizational Commitment on Employee Performance: Implications for Human Resource Strategy. 
Interdisciplinary Journal of Contemporary Research in Business, 3(11), 248-255.

Radosavljevic, Z., Cilerdzic, V., \& Dragic, M. (2017). Employee Organizational Commitment, International Review, 1-2, 18-26. https://doi.org/10.5937/intrev1702018R

Scholl, R. W. (1981). Differentiating commitment from expectancy as a motivating force. Academy of Management Review, 6, 589-599. https://doi.org/10.5465/amr.1981.4285698

Sethi, U. J. (2014). Influence of Work life balance on organizational commitment: A comparative study of women employees working in public and private sector banks, European Journal of Business and Management, 6(34), 215-219.

Uygur, A. (2007). Örgütsel bağlılık ile işgören performansı ilişkisini incelemeye yönelik bir alan araştırması. Ticaret ve Turizm Ĕgitim Fakültesi Dergisi, 1, 71-85.

Vardi, Y., Wiener, Y., \& Popper, M. (1989). The value content of organizational mission as a factor in the commitment of members. Psychological Reports, 65, 27-34. https://doi.org/10.2466/pr0.1989.65.1.27

Wiener, Y. (1982). Commitment in organizations: A normative view. Academy of Management Review, 7, 418-428. https://doi.org/10.5465/amr.1982.4285349

Wiener, Y., \& Vardi, Y. (1980). Relationships between job, organization, and career commitments and work outcomes - An integrative approach. Organizational Behavior and Human Performance, 26, 81-96. https://doi.org/10.1016/0030-5073(80)90048-3

Williams, L. J., \& Anderson, S. E. (1991). Job satisfaction and organizational commitment as predictors of organizational citizenship and in-role behavior. Journal of Management, 17, 601-617. https://doi.org/10.1177/014920639101700305

Yıldız, S., Savcı, G., \& Kapu, H. (2014). Motive edici faktörlerin çalışanların iş performansına ve işten ayrılma niyetine etkisi. (The Effect of Motivational Factors on Job Performance and Turnover Intention). Yönetim ve Ekonomi Dergisi, 21(1), 233- 249. https://doi.org/10.18657/yecbu.75006

\section{Copyright Disclaimer}

Copyright for this article is retained by the author(s), with first publication rights granted to the journal.

This is an open-access article distributed under the terms and conditions of the Creative Commons Attribution license (http://creativecommons.org/licenses/by/4.0/). 